\title{
Anti-Vascular Endothelial Growth Factor (Bevacizumab) Therapy Reduces Hypertrophic Scar Formation in a Rabbit Ear Wounding Model
}

\author{
Do Hoon Kwak, Tae Hui Bae, Woo Seob Kim, Han Koo Kim \\ Department of Plastic and Reconstructive Surgery, Chung-Ang University Hospital, Chung-Ang University College of Medicine, Seoul, Korea
}

Background Hypertrophic scarring is a pathological condition that occurs after trauma or surgery. Angiogenesis occurs more often with hypertrophic scarring than with normotrophic scarring. The regulation of angiogenesis is one of the key factors in hypertrophic scar management. Vascular endothelial growth factor (VEGF) is an essential factor in the angiogenetic response. This study investigated whether decreasing the level of VEGF is effective for treating hypertrophic scarring.

Methods Ten 8-week-old female New Zealand white rabbits were included. Four defects were created on each ear by using a 6-mm punch. Bevacizumab (Avastin, Roche Pharma, Basel, Switzerland) was administered in one ear and normal saline was administered in the other ear. Treatment was administered starting on day 2, every 2 days, until day 14. The levels of VEGF were measured using enzyme-linked immunosorbent assay on day 10 and histologic results were analyzed on day 40.

Results Bevacizumab induced-defects showed less hypertrophic scarring when compared with the control group as measured by the scar elevation index (SEI) and loose collagen arrangement. The SEl in the experimental group was $1.89 \pm 0.13$, compared to $1.99 \pm 0.13$ in the control group $(n=30, P=0.005)$. Additionally, the VEGF level was lower $(38.72 \pm 11.03 \mathrm{pg}$ vs. $82.50 \pm 21.64 \mathrm{pg}, \mathrm{n}=10, \mathrm{P}=0.001)$ and fewer vessels existed ( $8.58 \pm 0.76$ vs. $7.2 \pm 1.20$, $\mathrm{n}=10, \mathrm{P}=0.007$ )

Conclusions Preventing excessive angiogenesis is effective for preventing scar formation, especially with hypertrophic scarring. Although it is not an approach that is sufficient alone for the management of scarring, it may be one of several important strategies for scar treatment.

Keywords Cicatrix, hypertrophic / Neovascularization, pathologic / Bevacizumab / Angiogenesis inhibitors
Correspondence: Tae Hui Bae Department of Plastic and Reconstructive Surgery, Chung-Ang University Hospital, Chung-Ang University College of Medicine, 102 Heukseok-ro, Dongjak-gu, Seoul 06973, Korea

Tel: +82-2-6299-1615

Fax: +82-2-825-9880

E-mail: psbth@cau.ac.kr
No potential conflict of interest relevant to this article was reported.

\section{INTRODUCTION}

Hypertrophic scarring is one of the main issues of concern after trauma, surgery, or burns. In addition to the aesthetic consequences of hypertrophic scarring, itching sensation, pain, and contracture are also important problems that require attention. 
Many physicians have sought to manage hypertrophic scarring, and recently accepted treatments include steroid injection, radiotherapy, laser treatment, and topical silicone gel [1]. Owing to the complexity of the hypertrophic scar formation process and the many factors involved, each strategy of scar care is focused on a certain step in the hypertrophic scar formation process.

Angiogenesis is essential for wound healing and tissue regeneration, especially in the proliferative phase of healing. In the early stages of the wound healing process, robust neovascularization is observed. It is widely accepted that inflammation and delayed wound healing are related to scarring. Although angiogenesis is critical for wound healing, many studies have shown that an excessive angiogenic response causes scar formation $[2,3]$. Microvascular formation is more vigorous in hypertrophic scars than in normotrophic scars. Fibrotic diseases such as pulmonary fibrosis, with excessive angiogenesis, also show a relationship with fibrotic scar formation $[4,5]$.

Vascular endothelial growth factor (VEGF) is a key factor in angiogenesis. It is a homodimeric glycoprotein that regulates the permeability of blood vessels and tumor neovascularization. In pathologic scars, the expression of VEGF and the density of blood vessels are higher than in normal skin [6,7]. VEGF is related to fibrosis and inflammatory disease. In past studies, the concentration of VEGF has been shown to increase with fibrotic ocular disease, rheumatoid disease, and Crohn disease [8,9].

Bevacizumab (Avastin, Roche Pharma, Basel, Switzerland) is an anti-VEGF-A monoclonal antibody. It is the first angiogenesis inhibitor approved by the U.S. Food and Drug Administration for the prevention of tumor growth and it is widely used in the management of diabetic retinopathy due to fibrosis following neovascularization. In ophthalmologic departments, many studies are being conducted on the anti-angiogenetic effect of bevacizumab in vivo and in vitro $[10,11]$. Previous studies have demonstrated that anti-VEGF drugs were effective in managing scar formation $[12,13]$. This study was designed to elucidate the effects of anti-VEGF drugs on hypertrophic scars, which are characterized by high VEGF levels and a high number of blood vessels.

\section{METHODS}

This study was approved by the local Animal Review Committee (2016-00061). A total of 10 female, 8-week-old New Zealand white rabbits $(2,400-2,600 \mathrm{~g})$ were used, since rabbit ear models of hypertrophic scarring are widely accepted as research models [14].

\section{Procedure}

Rabbits were anesthetized by intramuscular injection of tiletamine/zolazepam (10 mg/kg; Virbac Korea, Seoul, Korea) and xylazine hydrochloride ( $2 \mathrm{mg} / \mathrm{kg}$, Rompun, Bayer Korea, Seoul, Korea) into the thigh. Including the perichondrium, four defects were created for each ear using a 6-mm punch biopsy. Remnant perichondrium was removed with a sharp blade until bare cartilage was exposed. Each ear was covered with Tegaderm (3M, St. Paul, MN, USA) after creation of the defects. One ear was used as the control and the other ear was used as the experimental ear in which bevacizumab was injected.

Bevacizumab injections were started on day 2 and administered every 2 days until day 14 . A dose of $0.2 \mathrm{~mL} / 5 \mathrm{mg}$ of bevacizumab was injected into each defect of the experimental ear. Normal saline, of the same volume as that of the bevacizumab injected in the experimental ear, was injected into the defects of the control ear. The injection was performed using an insulin syringe for minimal needling injury. Bevacizumab and normal saline were administered into the subcutaneous border of the defects. A total of 2 injections were performed into the opposite side of the defect each day and the injection sites were rotated $90^{\circ}$ on the next day.

One of 4 defects was harvested on day 10 for analysis of VEGF levels. The remaining defects were harvested on day 40 for evaluation of the scar elevation index (SEI), collagen arrangement, and number of vessels. An 8-mm punch was used to harvest tissue, including surrounding normal tissue.

\section{Enzyme-linked immunosorbent assay for the determination of VEGF levels}

The tissue harvested on day 10 was first dissected and washed with phosphate-buffered solution. Proteins were extracted with radioimmunoprecipitation assay buffer (Dynebio, Seoul, Korea) and centrifuged at 10,000 rpm for 10 minutes. The supernatant was used to measure VEGF levels in picograms by utilizing a rabbit VEGF enzyme-linked immunosorbent assay (ELISA) kit (NeoBiolab, Woburn, MA, USA). The microtiter plate of this kit is coated with a VEGF-specific antibody. Samples were co-incubated in wells along with a VEGF-horseradish peroxidase (HRP) conjugate. VEGF competed with the VEGFHRP conjugate for binding to the plate-bound antibody in the samples. Higher levels of VEGF in the samples led to decreased VEGF-HRP conjugate binding and a reduced signal. Captured VEGF-HRP was then quantitatively detected.

\section{Scar elevation index}

The histologic specimen was determined from a central portion of harvested defects, fixed in 10\% formalin, and stained using 
hematoxylin and eosin. The ratio of tissue height in the total wound area to the area of normal tissue below the hypertrophic scar, SEI, was calculated using a digital imaging program (Image J, National Institutes of Health, Bethesda, MD, USA) [15].

\section{Vascular analysis}

A total of 5 sections of each defect were randomly selected for counting the number of vessels. The specimen was immunostained using anti-CD31 antibody (ab199012, Abcam, Cambridge, UK). Using Image J, a pathologist counted the number of capillary blood vessels at a high magnification $(\times 400)$. According to criteria established by Weidner [16], blood vessels with a minor axis diameter of $50 \mu \mathrm{m}$ or narrower were regarded as capillary blood vessels. Previously existing large vessels were ignored. The mean numbers of blood vessels in the 5 sections were obtained.

\section{Scar color}

Two scars were selected in the same position from each ear. We categorized the scars by a scar color scale from points 1 to 4 . Er- ythematous skin color is induced by vessel conditions such as vessel dilatation, thin skin thickness, and vessel overgrowth.

\section{Statistical analyses}

The SEI, number of vessels, scar color scale, and VEGF levels were analyzed using SPSS ver. 19 (IBM Corp., Armonk, NY, USA). Comparisons of the experimental and control groups were performed using the Mann-Whitney $U$ test. A value of $\mathrm{P}<0.05$ was considered to be statistically significant. All results showed mean and standard deviation data.

\section{RESULTS}

\section{Histologic findings}

All wounds healed and epithelialized 2 weeks after the defects were created. Wound healing time was not significantly different between the 2 groups. Hypertrophic scars in the experimental group were much paler and less apparent than those in the control group (Fig. 1). The scar color in the experimental group was less erythematous (Fig. 2). Hematoxylin and eosin staining

\section{Fig. 1. Morphologic findings}

The scar was less visible and erythematous in the experimental group (A) compared to the control group (B).
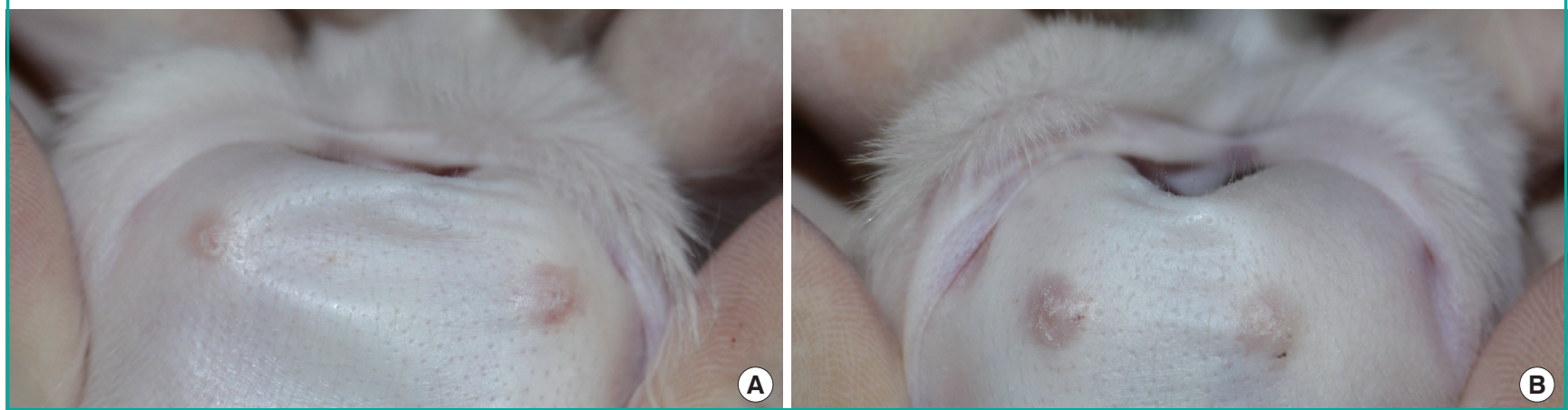

Fig. 2. Comparison of scar colors

Two defects were selected in the same position. Compared to the control ear defect, the experimental group had less erythematous scarring $(n=20, P<0.001)$.

\begin{tabular}{|c|c|c|}
\hline & Scale & Features \\
\hline & 1 & Not erythematous \\
\hline & 2 & Mildly erythematous \\
\hline & 3 & Moderately erythematous \\
\hline & 4 & Severely erythematous \\
\hline
\end{tabular}

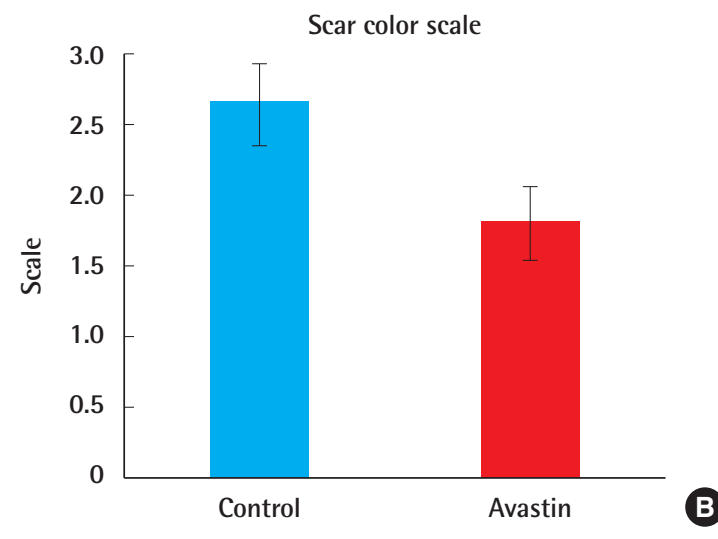




\section{Fig. 3. Histologic findings of the scar surface}

Compared to the control group (A), the experimental group (the anti-VEGF-treated group) showed less irregular and more flat scars (B). Vigorous angiogenesis was shown in the control defect (black arrow) (A, B: H\&E, $\times 100)$. VEGF, vascular endothelial growth factor.
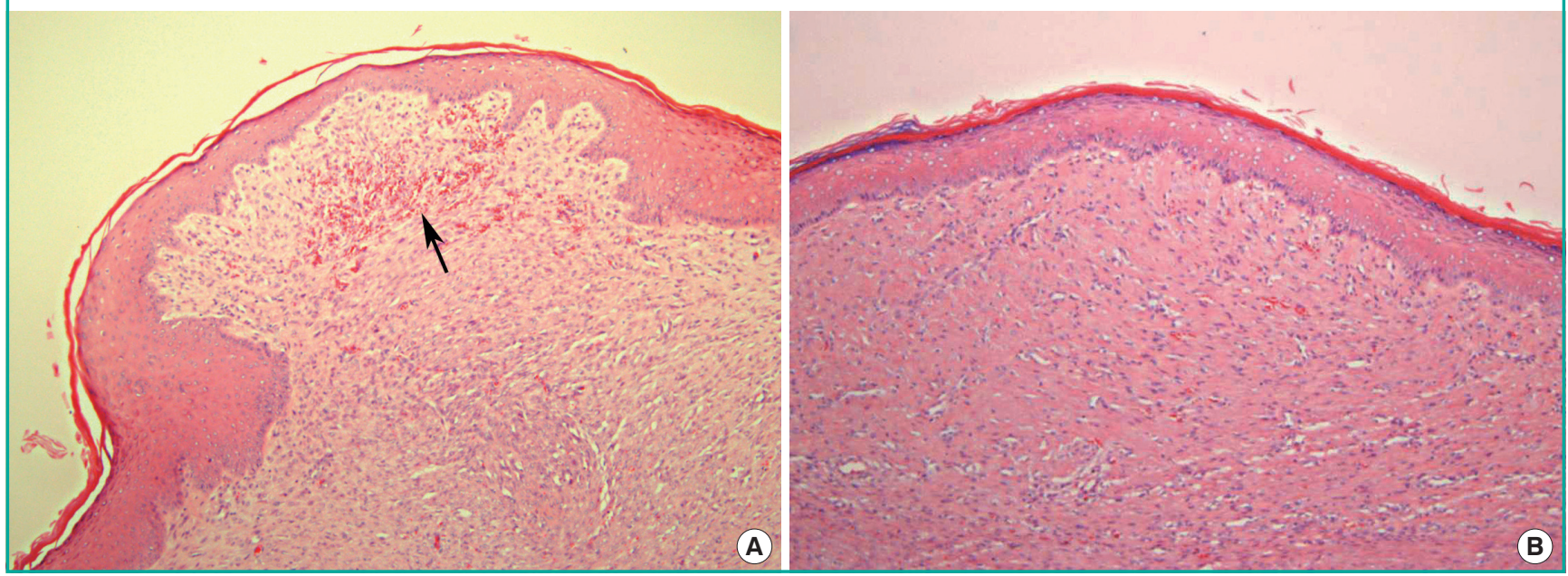

Fig. 4. Histologic findings of collagen distribution

Experimental groups showed regular and loose collagen distribution (A), whereas the control group showed dense and tangled collagen fibers (B). (A, B: Masson trichrome staining, $\times 400)$.

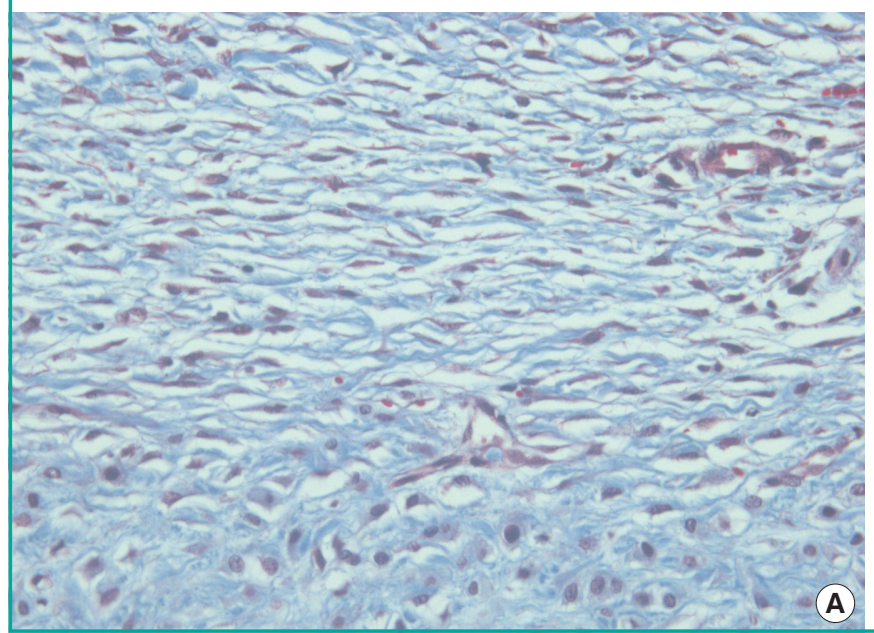

showed that scar elevation was lower and the scar surface was less irregular in the experimental group than in the control group (Fig. 3). The experimental group showed regular and loose collagen distribution, whereas the control group showed dense and tangled collagen fibers (Fig. 4).

\section{Scar elevation index}

The SEI was lower in the experimental group than in the control group $(\mathrm{n}=30, \mathrm{P}=0.005)$ (Fig. 5). The SEI in the experimental group was $1.89 \pm 0.13$, compared to $1.99 \pm 0.13$ in the control group.

\section{ELISA for the determination of VEGF levels}

On day 10, one of the defects was harvested to determine VEGF

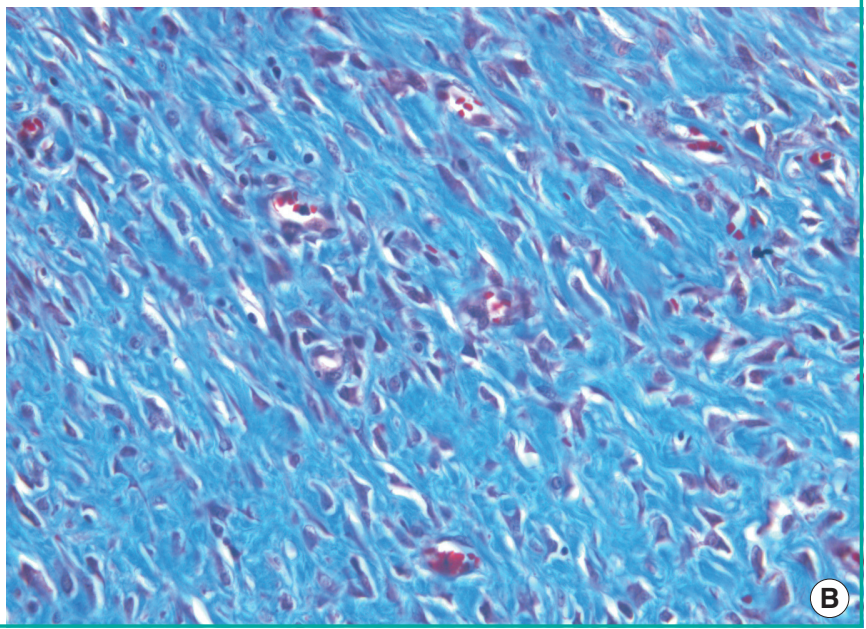

levels, for which ELISA was used. The level of VEGF in the experimental defects $(38.72 \pm 11.03 \mathrm{pg})$ was significantly lower than that in the control defects $(82.50 \pm 21.64 \mathrm{pg}, \mathrm{n}=10, \mathrm{P}=$ 0.001) (Fig. 6).

\section{Vascular phenotypes}

Harvested scars on day 40 were immunochemically stained using anti-CD31 antibody to detect endothelial cells. Five randomly selected histologic samples were analyzed by a pathologist using an imaging program. The average counts from the 5 fields $(\times 400)$ were used for analysis. The difference in the number of vessels between the 2 groups was significant ( $8.58 \pm 0.76$ vs. $7.2 \pm 1.20, n=10, P=0.007$ ) (Fig. 7). Moreover, overall endothelial cell distribution was denser in the control group (Fig. 8). 


\section{Fig. 5. Scar elevation index}

The scar elevation index measured using Image J software. The scar elevation index in the control group was $1.99 \pm 0.13$, compared to $1.89 \pm 0.13$ in the experimental group. The difference is statistically significant $(n=30, P=0.005)$.

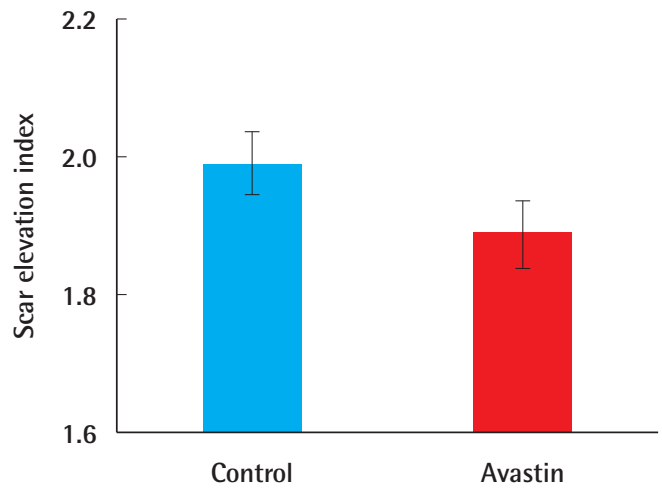

\section{Fig. 6. The level of VEGF}

The level of VEGF in the injected defects $(38.72 \pm 11.03 \mathrm{pg})$ was significantly lower than that in the control defects $(82.50 \pm 21.64 \mathrm{pg}$, $n=10, P=0.001)$. VEGF, vascular endothelial growth factor.

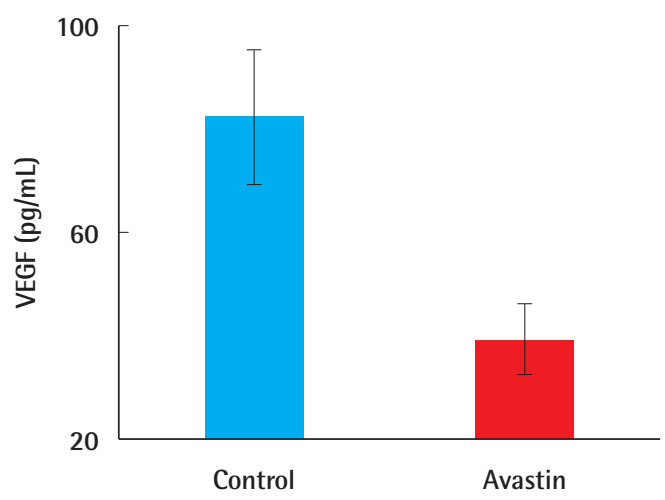

\section{DISCUSSION}

Many factors are involved in hypertrophic scar formation, and therefore they should be managed with treatments that take multiple approaches. There is no universal solution for scar treatment, rather than management of the scar. Currently used options, including steroid injection, pressure sheets, and operative revision, have drawbacks such as systemic problems, long treatment periods, and recurrence. Using many options at once or subsequently reduces drawbacks and enhances the effects. This study suggests another, additional option for scar management that can be used in conjunction with other methods.

Bevacizumab prevents angiogenesis and therefore has been used in the treatment of diabetic retinopathy. The formation of new surplus vessels triggers fibrosis in the retina. Because scar-

\section{Fig. 7. Comparison of the number of vessels}

A comparison of the number of vessels using immunochemical staining (CD31). The number of vessels in the experimental group is significantly lower than that in the control group (7.2 \pm 1.20 vs. $8.58 \pm 0.76, n=10, P=0.007)$.

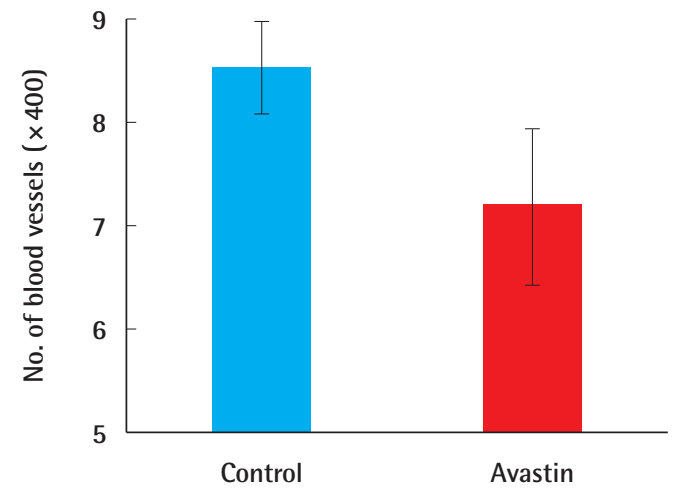

\section{Fig. 8. Endothelial cell distribution}

The overall endothelial cell distribution ( $A, B$ : determined via immunohistochemical staining with CD31, $\times 40)$ in the experimental group (A) is less dense than that in the control group (B). An apparent stained endothelial cell at low magnification (arrows).
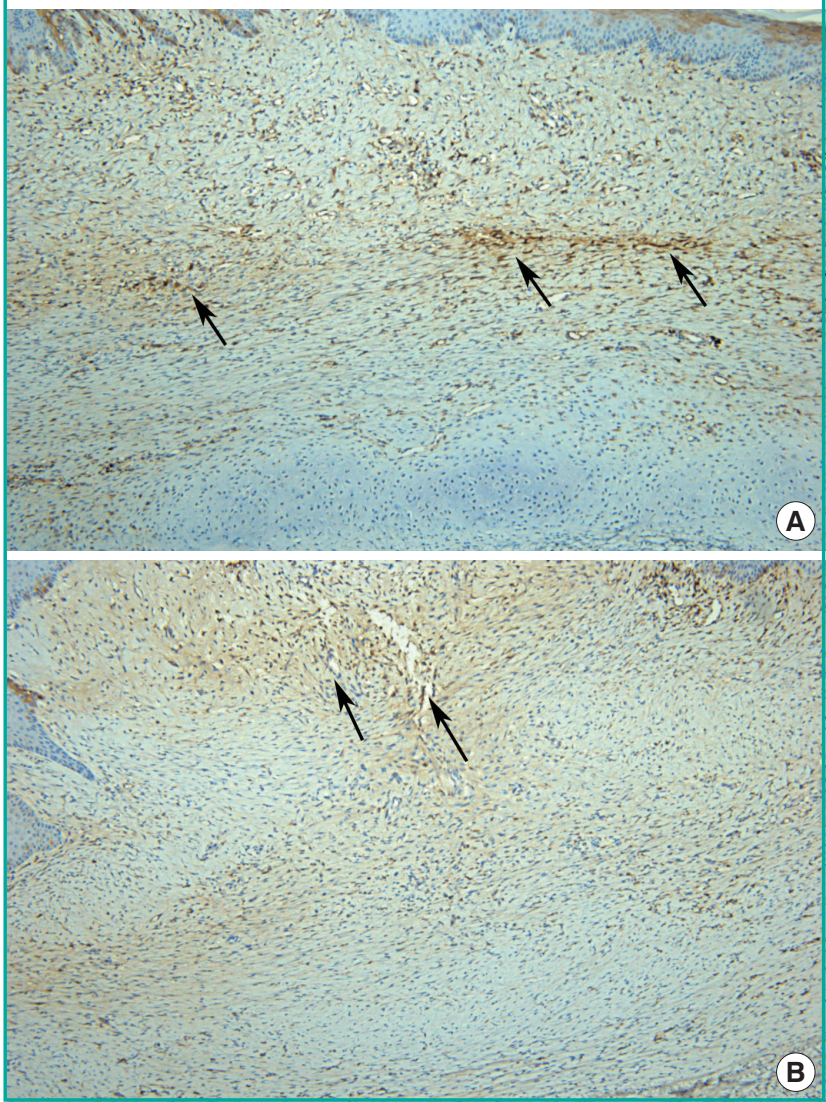

ring is a fibrotic process, bevacizumab can also be effective for preventing scarring. Numerous studies have demonstrated that anti-VEGF drugs reduce scar formation $[2,13]$. Scar formation 
is a normal process in the wound healing stage, but hypertrophic scarring and keloid are pathologic conditions that develop after wound healing. This experiment was designed to show the effects of an anti-VEGF drug on hypertrophic scar formation rather than on normal scar formation. Many studies have discovered the difference between normal scars and hypertrophic scars in relation to angiogenesis [3]. When excessive angiogenesis occurs, hypertrophic scars show high levels of VEGF $[6,7]$.

One problem associated with hypertrophic scars is the aesthetic aspect, specifically the erythematous color caused by increased blood flow. The findings showed that the anti-VEGF injected defects were less visible and erythematous. Perfusion of microcirculation was shown to decrease in another study evaluating the effects of anti-VEGF treatment [17]. Compared to other scar management options, anti-VEGF therapy may have strong effects on erythematous scar color.

The obvious difference in normal scars and hypertrophic scars is the degree of elevation. The lower SEI of anti-VEGF-injected defects shows that anti-VEGF therapy reduced scar thickness. The level of VEGF is high in many fibrotic diseases. The role of VEGF in fibrosis is not obvious, but VEGF must influence fibrosis. Moreover, in this study, Masson trichrome-stained slides of the control group showed dense and disordered collagen arrangements.

In many studies conducted on the effect of bevacizumab on rabbits, dosages of bevacizumab varied from 1.25 to $3.75 \mathrm{mg}$. In particular, previous experiments with subconjunctival injection of bevacizumab showed that $3.75 \mathrm{mg}$ of bevacizumab was effective for preventing angiogenesis and did not interfere with corneal epithelization or wound healing [18]. Since no study has been performed concerning the durability difference between skin and intraocular tissue, we supposed that skin is more durable than intraocular tissue, since skin is a normal barrier. For this reason, a dosage of bevacizumab that is higher than in previous conventional studies was used in this study.

Angiogenesis is crucial for wound healing. Although no significant wound healing time difference was shown between the experimental group and the control group in this study, antiVEGF induces delayed wound healing. We expect that the ideal effect of anti-VEGF will be achieved with suppressing overgrowth of new vessel formation, while not inhibiting the process of wound healing, which mainly depends on its concentration. Further research should investigate the relationship between concentration and effects.

Bevacizumab was originally used intravenously for oncological treatment or topically on the retina for diabetic retinopathy. In this study, in order to identify the effect of local injection, scar tissue was harvested in the middle of the injection period, at day
10. Local injection of bevacizumab is effective at lowering the level of VEGF, as shown in the ELISA analysis. VEGF concentrations and the number of vessels in the experimental group were both shown to be lower than in the control group. In a study on methods of application of bevacizumab, not only intravitreal application but also subconjunctival injection was effective [10]. Although the methods of application for bevacizumab are various, local injection is more effective for the prevention of scar formation in the early wound healing stage in relation to angiogenesis.

While additional studies will be needed to determine the mechanism, anti-VEGF treatment is effective in reducing hypertrophic scar formation. Recently, diverse scar treatments have been applied, such as steroid injections, silicon sheets, laser therapy, and surgery. However, the ideal treatment is prevention. To achieve a scar-free effect, the direction of scar management should be focused on wound-healing steps. Scar formation is related to many factors such as environment, race, underlying diseases, and trauma mechanisms, and therefore, multiple treatment approaches should be considered. This study suggests that bevacizumab may be used as an effective treatment option when deploying multi-directional scar treatments, for treatment of scar features, texture, and color.

Many studies and reviews have already revealed that antiVEGF treatment may be effective at preventing scar formation. But there are no studies about its application to a hypertrophic scar model, which features vigorous angiogenesis. Moreover, this experiment targeted early wound healing stages via direct injection for enhancing the efficacy of anti-VEGF therapy. The results of this study demonstrated the likelihood of the practical application of anti-VEGF for scar prevention.

Although bevacizumab reduces scar formation, it does have adverse effects. No research on the effect of local injection or topical application of bevacizumab to scars has been published. Further research should be performed in vivo to ensure the use of bevacizumab without adverse effects and to reveal the mechanisms underlying its effect.

\section{REFERENCES}

1. Ogawa R. The most current algorithms for the treatment and prevention of hypertrophic scars and keloids. Plast Reconstr Surg 2010;125:557-68.

2. DiPietro LA. Angiogenesis and scar formation in healing wounds. Curr Opin Rheumatol 2013;25:87-91.

3. van der Veer WM, Niessen FB, Ferreira JA, et al. Time course of the angiogenic response during normotrophic and hypertrophic scar formation in humans. Wound Repair Re- 
gen 2011;19:292-301.

4. Kalluri R, Sukhatme VP. Fibrosis and angiogenesis. Curr Opin Nephrol Hypertens 2000;9:413-8.

5. Wynn TA. Common and unique mechanisms regulate fibrosis in various fibroproliferative diseases. J Clin Invest 2007;117:524-9.

6. Bock O, Schmid-Ott G, Malewski P, et al. Quality of life of patients with keloid and hypertrophic scarring. Arch Dermatol Res 2006;297:433-8.

7. Ong CT, Khoo YT, Tan EK, et al. Epithelial-mesenchymal interactions in keloid pathogenesis modulate vascular endothelial growth factor expression and secretion. J Pathol 2007;211:95-108.

8. Danese S. Inflammation and the mucosal microcirculation in inflammatory bowel disease: the ebb and flow. Curr Opin Gastroenterol 2007;23:384-9.

9. Maruotti N, Cantatore FP, Crivellato E, et al. Angiogenesis in rheumatoid arthritis. Histol Histopathol 2006;21:557-66.

10. Nomoto H, Shiraga F, Kuno N, et al. Pharmacokinetics of bevacizumab after topical, subconjunctival, and intravitreal administration in rabbits. Invest Ophthalmol Vis Sci 2009; 50:4807-13.

11. Sinapis C, Routsias J, Sinapis D, et al. Pharmacokinetics of intravitreal bevacizumab (Avastin) in rabbits. Invest Ophthalmol Vis Sci 2010;51:2440.
12. Wang J, Chen H, Shankowsky HA, et al. Improved scar in postburn patients following interferon-alpha2b treatment is associated with decreased angiogenesis mediated by vascular endothelial cell growth factor. J Interferon Cytokine Res 2008;28:423-34.

13. Wilgus TA, Ferreira AM, Oberyszyn TM, et al. Regulation of scar formation by vascular endothelial growth factor. Lab Invest 2008;88:579-90.

14. Morris DE, Wu L, Zhao LL, et al. Acute and chronic animal models for excessive dermal scarring: quantitative studies. Plast Reconstr Surg 1997;100:674-81.

15. Ko JH, Kim PS, Zhao Y, et al. HMG-CoA reductase inhibitors (statins) reduce hypertrophic scar formation in a rabbit ear wounding model. Plast Reconstr Surg 2012;129:252e262e.

16. Weidner N. Chapter 14. Measuring intratumoral microvessel density. Methods Enzymol 2008;444:305-23.

17. Ren HT, Hu H, Li Y, et al. Endostatin inhibits hypertrophic scarring in a rabbit ear model. J Zhejiang Univ Sci B 2013; 14:224-30.

18. Mello GR, Pizzolatti ML, Wasilewski D, et al. The effect of subconjunctival bevacizumab on corneal neovascularization, inflammation and re-epithelization in a rabbit model. Clinics (Sao Paulo) 2011;66:1443-50. 